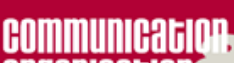
Organllotid|
Communication et organisation

Revue scientifique francophone en Communication organisationnelle

$52 \mid 2017$

Communauté, sociabilité et bien commun: approche internationale

\title{
Une communauté scientifique en construction : la recherche en Communication Organisationnelle, France et Brésil
}

The constitution of a scientific community: Organizational Communication research in France and Brazil

Una comunidad científica en construcción: investigación en Comunicación

Organizacional en Francia y Brasil

Uma comunidade científica em construção: pesquisa em Comunicação

Organizacional na França e no Brasil

Alice Zozima Paris Rego de Souza

\section{OpenEdition}

\section{Journals}

Édition électronique

URL : https://journals.openedition.org/communicationorganisation/5640

DOI : 10.4000/communicationorganisation.5640

ISBN : 979-10-300-0257-7

ISSN : $1775-3546$

Éditeur

Presses universitaires de Bordeaux

Édition imprimée

Date de publication : 1 décembre 2017

Pagination : 35-46

ISBN : 979-10-300-0163-1

ISSN : 1168-5549

Référence électronique

Alice Zozima Paris Rego de Souza, « Une communauté scientifique en construction : la recherche en Communication Organisationnelle, France et Brésil », Communication et organisation [En ligne], 52

2017, mis en ligne le 01 décembre 2020, consulté le 02 janvier 2022. URL : http://

journals.openedition.org/communicationorganisation/5640 ; DOl : https://doi.org/10.4000/

communicationorganisation. 5640 


\title{
Une communauté scientifique en construction: la recherche en Communication Organisationnelle, France et Brésil
}

\author{
Alice Zozimo Paris Rego de Souza ${ }^{1}$
}

\section{Introduction ${ }^{2}$}

Le numéro 52 de la revue Communication E Organisation nous invite à discuter et à réfléchir sur ce que peut être ou devenir une Communauté et plus précisément une communauté scientifique. Dans cet article, nous proposons quelques pistes pour réfléchir au processus de construction d'une Communauté Scientifique en Communication Organisationnelle, qui ne soit pas seulement nationale mais élargie, en nous focalisant sur les rapports entretenus entre chercheurs français et brésiliens. Nous pourrions pour cela poser qu'existent plusieurs niveaux dans les formes d'échanges scientifiques : (1) les chercheurs se lisent et leurs textes portent les marques de leurs références (citations); sachant ainsi que la lecture est un élément de la pratique scientifique individuelle : il y a ainsi des "passeurs ». (2) Que des associations, des revues, organisent des échanges : il y a aussi des « organisateurs ». Dès lors des rencontres, un agenda partagé permettent que des collectifs se construisent. Cette organisation peut concerner bien des communautés (de savoirs, de pratiques) car

sans interactions sociales, une idée reste lettre morte. Pour se développer et atteindre une certaine maturation, une idée doit être exposée à un contexte participatif. Plus ce dernier sera riche en interactions sociales et en nombre de participants, plus il sera possible d'interconnecter les idées entre elles au sein de communautés d'apprentissage ou de pratiques en réseau sous lesquelles les participants pourront échanger des points de vue, les analyser, les approfondir, les partager et se les approprier en collectivité... (Scardamalia ${ }^{3}$ et Boreiter 2000).

1 ATER à l'Institut d'Administration et de Gestion de l'Université de Toulon, docteur en Sciences de l'Information et de la Communication à l'Université de Toulon en cotutelle à l'École des Communications et Arts de l'Université de São Paulo (ECA-USP / PPGCOM), alice_zozima@hotmail.com

2 Je remercie ici l'aide du Professeur Pierre Delcambre dans cet article pour avoir fait un travail d'éclairage et de montage d'idées.

3 Marlene Scardamalia est professeure en éducation et recherche de l'Université de Toronto. 
Lors de ces échanges, nous nous écoutons, nous apprenons des noms, nous commençons à nous «reconnaître». (3) Consciente des limites (linguistiques, géographiques, communicationnelles mais aussi, peut-être, cognitives), nous avançons l'hypothèse selon laquelle une communauté scientifique est possible en communication organisationnelle.

Les communautés de chercheurs en communications organisationnelles sont-elles nationales? Ou dotées d'échanges bilatéraux ou internationaux? Si nous acceptons l'idée que ce sont des formes de socialisation qui construisent ou défont des communautés, nous devenons plus attentifs aux pratiques et aux valeurs de ceux qui se reconnaissent comme membres d'une communauté. Or une communauté, bien sûr, nous " précède » (connaissance partagée de textes, moments, gens, anciens ou arrivants) mais aussi elle nous « excède » (elle est plus large que notre singularité). Dès lors il est utile de nous poser la question : dans la perspective de la construction d'une "Communauté Scientifique en Communication Organisationnelle franco-brésilienne», quelles valeurs et quelles histoires sont partagées?

Pour explorer cette question, nous procèderons en deux temps. D'abord, nous reprendrons quelques éléments d'une thèse récente portant sur les dynamiques françaises et brésiliennes de la construction de « disciplines professionnelles » (des métiers de la communication d'entreprise), de leurs théorisations, en nous arrêtant plus précisément sur la manière dont des formations et des recherches académiques se sont installées dans les deux pays. Les différences et les décalages temporels dans les processus " généalogiques » dans les deux pays en fait sont considérables. Dans un second temps nous reviendrons sur une enquête exploratoire que nous avions menée en démarrage de notre thèse: il s'agissait de documenter, si c'était possible, l'état des échanges entre chercheurs français et brésiliens dans ce champ, par une étude de leurs bibliographies. Ces deux premiers points nous permettront de reprendre en conclusion notre question: dans l'état des échanges des dix dernières années, sur quels points faut-il travailler plus pour soutenir les dynamiques qui renforceraient une communauté scientifique franco-brésilienne en "Communication Organisationnelle ». Nous souhaitons montrer les études, les perspectives et les avancements réalisés par ces deux communautés afin de connaître la possibilité de construire un savoir commun qui nous concerne.

\section{Métiers, formations et recherche en communication organisation- nelle en France et au Brésil}

Notre travail antérieur s'intéressait à la généalogie des pratiques professionnelles et des constructions théoriques (professionnelles et académiques), dans une comparaison Brésil-France à propos des " communications organisationnelles ", une thèse titrée "Le monde de la Communication Organisationnelle: Pratiques et Recherche. Une étude généalogique, France 
et Brésil» (Paris R. S. 2017). Toutefois, dans cette thèse, la question «d'une communauté en construction", en France et au Brésil, ou dans un champ géographique plus large (question de ce numéro 52) était moins traitée que celle des dynamiques d'installation de métiers, de formations et de recherches. Nous nous sommes intéressée aux périodes d'installation et de formalisation de pratiques professionnelles (Relations Publiques, puis Communication, Communication d'Entreprise, Communication Organisationnelle, jusqu'à la "Communication Intégrée »). Pour envisager ces histoires et particulièrement celles des théories et doctrines produites par des professionnels et des universitaires, il nous a fallu faire un travail d'exploration; établir un corpus fut inévitable, nous avons délimité les sources à partir de publications (revues, journaux et site web) ${ }^{4}$ référentes, spécialisées dans la Communication Organisationnelle. Nous avions en effet l'intention de comprendre d'où venaient les racines de ce champ de recherche.

Remonter aux sources et aux traditions des chercheurs actuels nous amenait à faire, pays par pays, des généalogies. Nous étions attentive aussi bien aux professionnels réputés qui ont commencé à formaliser leurs pratiques et à les enseigner, ainsi certains devenant des universitaires, démarrant la recherche académique dans un contexte « disciplinaire ». Pour travailler cette perspective généalogique et comparative, nous nous sommes appuyée sur le concept d'Institutionnalisation, avec les deux concepts-clefs travaillés par Robert Boure dans son étude de l'Histoire des Sciences Sociales en France (Boure 2007): l'Institutionnalisation Cognitive caractérisée par la formulation des questions de recherche, des concepts et des théories, ainsi que par le travail sur les méthodes et sur la délimitation du champ de savoir, le choix des objets et des terrains. Un travail parfois réalisé par des contributions individuelles, mais aussi par des groupes de chercheurs. Et l'Institutionnalisation Sociale qui correspond à la mise au point de modes d'organisation de la recherche et de l'enseignement. C'est-à-dire, la création des laboratoires, des postes, des modes de financement et les systèmes de publications : l'institutionnalisation sociale est un phénomène de grande amplitude temporelle; elle a aussi des aspects économiques et elle est aussi très ancrée dans l'aire géographique, le plus souvent nationale, où des décisions se prennent.

Notre travail a ainsi permis de repérer des phénomènes fondamentaux pour comprendre que c'est «nationalement» que se sont construits, dans les deux pays considérés, les «champs de recherche» (France) ou «sous discipline» (Brésil) de la «Communication Organisationnelle».

À notre grande surprise, nous découvrions:

1. Qu'entre les deux pays il existe une temporalité très différente : d'un côté, au Brésil, les formations et les recherches se mettent en place dès les années 1960, avec la construction, dès 1985, sous le nom de

4 Ce repérage de publications fut fait pour la période de 2007-2015. 
« communication organisationnelle », d'un champ de recherche associé aux formations universitaires. En France les Sciences de l'Information et de la Communication (SIC) ne naissent qu'au milieu des années 70 et il faut attendre le milieu des années 90 pour qu'un groupe de recherche spécifique se mette en place sur les « communications organisationnelles ».

2. Que les chercheurs brésiliens entretiennent avec le monde professionnel des rapports (conseil, consultance, recherches) qui font partie d'une culture acceptée et transmise, alors qu'en France le rapport au monde économique des communications (communications d'entreprise, mais aussi agences) est plus compliqué, le contact privilégié étant celui consacré par les formations professionnelles universitaires.

3. Que, au-delà de la période d'installation, marquée par de grandes différences entre les deux pays, la période récente (2006-2016) est marquée par de grandes difficultés, des deux côtés de l'Atlantique, pour faire un état des lieux de ce champ, pour caractériser les différentes approches. Ici aussi des différences existent : nous ne pouvons pas repérer un référentiel commun.

4. Que le Brésil fut le pionnier en termes de recherche sur la Communication Intégrée: celle-ci était enseignée comme une approche dans la discipline de la Communication Organisationnelle, dans les Sciences de la Communication à l'Université de São Paulo ; aux États-Unis, elle existait aussi comme une discipline, mais traitée et liée au Marketing. Alors que de leur côté les chercheurs français ont construit un autre rapport de connaissance pratique avec leurs terrains et ne sont pas engagés dans cette étude : ils ont laissé des professionnels du marketing proposer un management intégratif.

Ainsi notre travail de thèse a montré la forte structuration nationale. Certes nous pouvions retrouver dans les deux pays une « influence américaine », aussi bien pour la mise en place des métiers (Relations Publiques, Journalisme d'Entreprise) que dans les approches théoriques universitaires (Speech Communication de Redding ${ }^{5}$ ); les références communes aux travaux américains (en Communication Organisationnelle et plus largement en "Communication ") semblent fonctionner comme un «tiers " médiateur ${ }^{6}$. Cette structuration nationale est comme un cadre pour le développement de chaque champ de recherche et nous pouvons comprendre dès lors que le

5 W. Charles Redding était directeur du Centre de Recherche de Communication (CRC) de l'Université Purdue, dans l'Indiana, USA. En tant que professeur de communication, il enseignait surtout l'argumentation du débat, l'art oratoire en anglais, la phonétique, l'interview, la persuasion, l'histoire du discours verbal et la Communication Organisationnelle.

6 Nous remercions Nicole D'Almeida pour cette suggestion. 
partage de valeurs, de mémoire, de discussions n'est pas seulement limité par l'état linguistique des échanges, mais aussi par une méconnaissance réciproque.

\section{Des communautés scientifiques et leurs bibliographies}

Nous avons ainsi montré le rôle majeur des phénomènes nationaux dans l'institutionnalisation sociale, et cognitive, de la Communication Organisationnelle, comme discipline et champ de recherche. Mais cela nous a aussi amenée à relever le rôle de multiples organisateurs (des personnalités, des revues, des collectifs, des associations) qui construisent des agendas, sollicitent des articles, organisent des échanges. C'est vraisemblablement l'état des organisations qui est actuellement retravaillé pour que des échanges plus nombreux, plus riches, plus discutants, se développent entre France et Brésil.

Dans ce second moment de notre article, avec une autre méthodologie, nous pourrions nous demander si les chercheurs concernés, par leurs pratiques scientifiques mêmes, contribuent à reconfigurer les échanges. En effet, si les lieux d'échange permettent de "savoir qui est qui ", le développement de recherches personnelles ou collectives que l'on veut inscrites dans un champ de recherche déterminé, provoque des choix de lectures et des décisions de références bibliographiques.

\section{La méthodologie}

En premier lieu nous avons fait un travail de repérage des revues, des associations et des groupes de chercheurs en Communication Organisationnelle en France et au Brésil. Nous nous sommes alors appuyée, pour les travaux français, sur une revue spécialisée du champ, la Communication \& Organisation et sur le site Archive Ouverte en Sciences de l'Information et de la Communication $\mathrm{du}$ Centre National de la Recherche Scientifique (Archivesic-CNRS ${ }^{7}$ ).

Pour la revue, nous avions trouvé une liste des auteurs français du domaine de la Communication Organisationnelle, réalisée en 2008 par Benoit Cordelier et Hélène Montagnac-Marie dans le numéro 33 de Communication E Organisation (Cordelier et Montagnac-Marie 2008). Dans cette liste nous avons relevé les auteurs, majoritairement - des professeurs et HDR français -, les plus cités dans les articles et les travaux publiés entre les années 2007 et 2014.

D’un autre côté, par une méthode moins centrée sur des auteurs repérés avec Archivesic, nous avons été accompagnée par Gabriel Gallezot ${ }^{8}$. Il fallait

7 L'Archivesic-CNRS est un espace francophone qui permet aux chercheurs en SIC de déposer des documents, textes, articles, chapitres de livres ou autres (Gallezot 2003).

8 Gabriel Gallezot est Maître de Conférences à l'UNS (Université Nice Sophia Antipolis), Membre du laboratoire I3M (EA3820 - Information Milieux Médias Médiation), Co-responsable de l'Unité Régionale de la Formation à l'Information Scientifique et Technique, et Membre du CNU 71e section. Il est aussi le cofondateur de l'Archive Ouverte : @rchiveSIC. Travail réalisé avec le CCSD (CNRS). 
d'abord distinguer une liste de publications des auteurs en SIC, car il n'existait pas encore une rubrique spécifique sur les publications en Communication Organisationnelle. Nous avons repéré environ 2000 documents mis en ligne. Nous avons réduit le corpus en plusieurs périodes pour aboutir à 82 documents, en ne retenant que des communications faites par les chercheurs en Communication Organisationnelle. À notre grande surprise quelques auteurs de notre liste initiale ne faisaient pas partie des documents analysés.

Pour la partie brésilienne, cette recherche des auteurs principaux de ce champ fut un peu plus simple, car il existe un Portail de la Production Scientifique en Sciences de la Communication, l'UniverCiencia.Org9', qui sépare par domaine les publications ; nous avons sélectionné 3771 articles publiés en Communication Organisationnelle. Nous avons aussi fait appel à l'Association Brésilienne de Chercheurs en Communication Organisationnelle et Relations Publiques (Associação Brasileira de Pesquisadores de Comunicação Organizacional e Relações Públicas - ABRAPCORP $\left.{ }^{10}\right)$ : de multiples informations utiles se trouvent dans leur site institutionnel. Nous avons agi de la même façon que pour la France, nous avons relevé les auteurs concernant majoritairement les professeurs qui avaient la "livre-docência » (équivalente au professeur HDR en France) et étaient plus cités dans les articles et les travaux publiés entre les années 2007 et 2014 toujours dans le même champ.

Nous avons décidé de restreindre notre analyse aux travaux qui faisaient référence à la Communication Organisationnelle. Enfin nous avons retenu la suivante liste par ordre alphabétique pour les chercheurs français : Alex Mucchielli, Arlette Bouzon, Catherine Loneux, Christian Le Moënne, Françoise Bernard, Gino Gramaccia, Jacques Bonnet, Michel Durampart, Nicole d'Almeida et Pierre Delcambre. Côté Brésil: Adriana Machado Casali, Angela Marques, Cláudia Peixoto Moura, Cleusa Maria Andrade Scroferneker, Ivone De Lourdes Oliveira, João José Azevedo Curvello, Margarida Maria Krohling Kunsch, Paulo Nassar, Rudimar Baldissera, Wilson Costa Bueno.

Nous n'avons finalement gardé que trois à cinq publications pour chaque auteur; c'est ainsi que nous avons approfondi notre connaissance institutionnelle des chercheurs français et brésiliens, des revues, des références et des citations qui étaient les leurs. En quelque sorte, nous mettions ainsi

9 Le Portail de la Production Scientifique en Sciences de la Communication, UniverCiencia.Org, est un portail brésilien disponible gratuitement en ligne où se trouvent des informations sur les articles de magazines, revues, thèses / mémoires, livres / chapitres, événements, matériel éducatif et d'autres produits publiés par la communauté scientifique et universitaire du domaine des Sciences de la Communication dans plusieurs pays, dans les langues suivantes: portugais, anglais, français et espagnol.

10 Lidée de la création de l'Abrapcorp, eut lieu en octobre 2005, au cours du premier Forum de Chercheurs Brésiliens en Communication Organisationnelle et Relations Publiques. À cette occasion un comité fut formé coordonné par Margarida Kunsch et les professeurs cités ci-dessus pour élaborer le statut de l'entité. Cette entité est autonome et comme les douze autres associations du secteur de communication, est liée à Socicom - Fédération Brésilienne des Associations Scientifiques et Académique de la Communication, créée en septembre 2008. 
au point une base bibliographique ouvrant à une temporalité plus longue où nous pouvions, en remontant le fil du temps, lire les sources des uns et des autres. Cela nous conduisait à une nouvelle investigation: un repérage des sources auxquelles se référaient les uns et les autres.

Ce premier pas nous a amenée à construire des tableaux à partir de chaque article dans le programme Excel. Nous avons créé les rubriques suivantes: auteurs, publication, numéro, nom d'article, sujets, université et/ ou laboratoire d'appartenance, discipline d'appartenance, nom de la revue, l'année de publication, référence bibliographique (dans cette rubrique, pour chaque auteur cité nous avons aussi vérifié les dates de publications), type de publication, langue, revue référencée et enfin l'auto-citation. Ces tableaux nous ont permis, avec l'aide du professeur Eric Boutin ${ }^{11}$ de construire des «toiles d'araignée ${ }^{12}$ » en utilisant le logiciel Netdraw (gratuit en ligne). Ces toiles d'araignée sont représentées visiblement dans notre étude (Paris. R. S., 2017).

\section{Un repérage de lectures présentes dans les bibliographies des chercheurs}

Notre premier objectif fut de comparer les citations et les références faites par les chercheurs français et les brésiliens. Cette étude bibliographique nous a permis de faire une analyse de données et à constater l'absence de citations ou de liens entre ces chercheurs comme le montrent les toiles d'araignées ci-dessous.

Voici le résultat en format de tableau et les analyses réalisées pour les publications des années 2007 à 2014 :

\begin{tabular}{|c|c|}
\hline Analyse numéro 1: & Analyse numéro 2: \\
\hline $\begin{array}{l}\text { Nous avons observé que dans l'analyse numéro } \\
1 \text { en ce qui concerne les chercheurs français il } \\
\text { n'existe aucune citation ou lien entre les auteurs } \\
\text { français et brésiliens. Cependant, nous vérifions } \\
\text { qu'il y a une très forte unité de citations entre } \\
\text { eux. C'est-à-dire des citations des groupes } \\
\text { internes. }\end{array}$ & $\begin{array}{l}\text { Dans un deuxième temps nous avons } \\
\text { cherché de comprendre si les chercheurs } \\
\text { français avaient l'habitude de citer des } \\
\text { auteurs étrangers ou dans leur propre langue. } \\
\text { Nous avons observé qu'il y a une forte } \\
\text { présence canadienne chez les Français. Nous } \\
\text { nous rendons compte que les liens (vers des } \\
\text { langues autres que le français) sont l'apanage } \\
\text { de quelques chercheurs. }\end{array}$ \\
\hline
\end{tabular}

11 Eric Boutin actuellement président de l'université de Toulon, est professeur des universités en SIC au sein du département Techniques de Commercialisation de l'IUT de Toulon. Il est aussi directeur de recherche sur les sciences du web au laboratoire I3M.

12 Dans un graphique en radar, aussi appelé « toile d'araignée », il y a autant d'axes que de catégories, tous les axes partent du point central. Les catégories sont autour du graphique (axe $\mathrm{X}$ ), les valeurs des séries à l'intérieur, des lignes droites relient les données d'une série en formant un polygone. Un graphique radar supporte plusieurs séries, chaque série étant identifiée par une couleur différente. 


\begin{tabular}{|l|l|}
\hline Analyse numéro 3: & $\begin{array}{l}\text { Analyse numéro 4: } \\
\begin{array}{l}\text { Nous avons observé que dans l'analyse numéro } \\
\text { i en ce qui concerne les chercheurs brésiliens } \\
\text { il existe une très forte interaction entre eux, } \\
\text { nous trouvons ainsi des références dans la } \\
\text { langue nationale. Cependant, n'est pas exclue } \\
\text { la présence des publications dans une langue } \\
\text { étrangère, principalement la nord-américaine. } \\
\text { observé les liens entre les chercheurs } \\
\text { brésiliens, les chercheurs français et les } \\
\text { chercheurs qui apparaissent à la fois dans } \\
\text { les deux corpus. Notre dernière analyse } \\
\text { traite des points communs entre les deux } \\
\text { communautés. Notre travail a enfin abouti à } \\
\text { quelque chose de bien plus approfondi et à la } \\
\text { prise d'une décision définitive sur la direction } \\
\text { de notre recherche. Nous analysons qu'il n'y a } \\
\text { pas beaucoupde citations entre les chercheurs }\end{array} \\
\text { français et brésiliens dans le domaine de } \\
\text { la Communication Organisationnelle. } \\
\text { Cependant, il existe un noyau commun } \\
\text { concernant quelques références citées par les } \\
\text { deux parties. Comme par exemple : Morin, } \\
\text { Bernays, Taylor, Putman, Mattelart, Castells, } \\
\text { Grunig, Morgan... }\end{array}$ \\
\hline
\end{tabular}

Nous pourrions dire globalement de notre étude que la France et le Brésil ne se sont jamais posé la question de l'interconnaissance scientifique entre eux, alors que par leurs bibliographies, les chercheurs français et les chercheurs brésiliens font toujours référence aux travaux nord-américains. Donc, cette étude nous a permis de vérifier que par « hasard » les approches nord-américaines étaient les plus « communes ", partagées. Est-ce logique ou étonnant, ce n'est pas le lieu de traiter cette question. Mais, comment expliquer que ces deux « communautés ", pour autant qu'elles existent, n'arrivent pas ou peu à communiquer de manière directe ? Une autre observation a priori tout aussi étonnante a été de constater que nombre de chercheurs confirmés sont absents des bibliographies des autres, aussi bien pour le Brésil que pour la France. Si les bibliographies montrent des liens de travail et pas seulement des « références ", cela pourrait montrer que le travail d'article ne s'appuie pas forcément sur une tradition reconnue mais plutôt sur la « mise au travail » de textes et d'auteurs.

\section{Pour conclure}

Le fait de distinguer, dans cet article, les lectures-bibliographies, les échanges organisés et l'interconnaissance et les collectifs ou communautés scientifiques nous a amenée à montrer l'importance des phénomènes organisationnels liés à l'institutionnalisation sociale, mais aussi cognitive, et la faiblesse numérique des liens individuels de référence, d'un côté et de l'autre de l'atlantique, d'où le rôle central de " passeurs ». 
Mais encore, notre étude, notamment dès lors que nous étudions la période récente des recherches en " communication(s) organisationnelle(s) », 20062016, nous a permis de voir l'effort énorme, mais aussi la grande difficulté des auteurs cherchant à faire des bilans, cherchant à caractériser les différentes approches repérables, cherchant enfin à indiquer les perspectives nouvelles, en cours. Travail énorme et difficulté de lecture et de connaissance de ses collègues, ainsi que de clairvoyance épistémologique pour " catégoriser " du vivant, proposer des interprétations sur des évolutions théoriques. Cette difficulté est en effet celle de faire des nomenclatures, des listes et de comprendre le jeu des théorisations multiples. Linda Putnam ${ }^{13}$ réorganise la nomenclature pour intégrer les interprétativistes (Putnam, Phillips et Chapman 1996, 375-408), Pierre Delcambre ${ }^{14}$ tente une analyse " générationnelle » (Delcambre 2008), Nicole d'Almeida ${ }^{15}$ avec Yanita Andonova ${ }^{16}$ s'essaie aussi, à la difficulté, de clarifier la diversité (d'Almeida et Andonova 2006). Même chose au Brésil avec Ivone de Lourdes Oliveira ${ }^{17}$ et Ângela Salgueiro Marques ${ }^{18}$ (Marques et Oliveira 2015, 109-124) et Margarida Maria Kroling Kunsch ${ }^{19}$ (Kunsch 2006 : 23-31). Cet aspect est pourtant essentiel pour alimenter les discussions, permettant ainsi des débats déjà mis en place de manière interne dans les groupes comme l'Org\&Co en France et l'Abrapcorp au Brésil.

13 Linda L. Putnam fait partie du Département de Communication de l'Université de Santa Barbara en Californie. Elle est très reconnue aujourd'hui pour ses recherches qui portent sur : la négociation et la gestion des conflits au sein des organisations, des études de discours d'organisation, des groupes et des équipes, et des études de genre dans les organisations.

14 Pierre Delcambre est professeur émérite en SIC à l'Université Lille 3 et membre de l'équipe Communications Organisationnelles et Processus d'Innovation (COPI) de GERiiCO. Il développait une analyse des pratiques et activités de communications ordinaires (communications de travail, communications publiques) d'entreprises et d'organisations (Travail social, Chantiers navals, Marine marchande, secteur culturel du spectacle vivant).

15 Nicole d'Almeida est professeure en sciences de l'information et de la communication au Celsa (Université de Paris-Sorbonne). Elle enseigne et dirige des formations et des recherches sur les thèmes suivants : communication interne et externe, récits organisationnels, dynamique de l'opinion, communication de l'environnement et développement durable, RSE.

16 Yanita Andonova est maître de Conférences à l'Université de Paris 13 où depuis 2015 elle est directrice adjointe de l'UFR Sciences de la Communication et chercheur au LabSIC.

17 Ivone de Lourdes Oliveira est professeure de Communication Sociale, et chercheure en Communication Organisationnelle à l'Université Catholique de Minas Gerais (PUC/MG), où elle enseigne le Master en 'Communications et les Interactions avec les Médias'. Elle a fait aussi un post-doctorat à l'Université de Toulouse - Paul Sabatier, en 2013, avec laquelle elle maintient des échanges jusqu’à présent.

18 Angela Cristina Salgueiro Marques est professeure de Communication Sociale, et chercheure en Communication Organisationnelle à l'Université Fédérale de Minas Gerais (UFMG). Elle a fait aussi un postdoctorat à l'Université Stendhal à Grenoble III en 2007 avec laquelle elle maintient des échanges jusqu'à présent.

19 Margarida Maria Krohling Kunsch est professeure HDR de l'École de Communication et Arts, de l'Université de São Paulo - ECA/USP. Membre de l'ABRAPCORP, elle est directrice des Relations Internationales de la Fédération Brésilienne des Associations Scientifiques et Académiques de Communication/Socicom. Elle a été deux fois présidente de la Société Brésilienne des Études Interdisciplinaires de la Communication/ INTERCOM et de l'Association Latino-américaine d'Investigation de la Communication/ALAIC. Elle est l'auteure entre autres de 38 livres. Plus de détail sur son parcours sur le lien : http://buscatextual.cnpq.br/ buscatextual/visualizacv.do?id=K4783236H1 
Il faut en outre noter que dans les deux pays, la socialisation, si importante pour la construction de communautés, est appuyée certes sur quelques valeurs communes, mais ne construit pas les mêmes mémoires, ni parfois les mêmes approches des phénomènes - nous devrions dire aussi des « gens » - à étudier. Cependant, si ces communautés nationales sont prêtes à créer des communautés internationales, notre question et avant tout : est-ce que les chercheurs de ces deux communautés savent ce qui est fait dans chaque pays d'interconnaissance, seraient-ils prêts à critiquer les travaux des uns et des autres? Est-il par exemple légitime d'être à la fois consultant et chercheur ? Qu'est-ce quêtre "critique "? Que valent les recherches visant à résoudre des problèmes précis et situés de professions ou du Management : sont-elles « fonctionnalistes » et donc d'un autre temps? Nous n'avons pas les réponses mais pouvons-nous oublier que ces questions scientifiques se posent, ici et là. Il est ainsi nécessaire de prendre conscience que les débats scientifiques sont plus malaisés dans des échanges internationaux.

Et pourtant, n'est-ce pas une des valeurs propres aux communautés scientifiques ?, pour reprendre les propos de Michel Dubois, dans son article « communauté scientifique » (dans Savidan et Mesure 2006) « être scientifique ne se résume jamais à la mise en œuvre d'une rationalité abstraite et solitaire (...) C'est également et surtout, après un long processus de socialisation, être membre d'un collectif orienté vers la production méthodique de connaissances, privilégiant la mise en commun des produits de l'activité comme la mise en pratique du principe de preuve publique ».

Donc, en revenant à la thématique de ce numéro 52, la question qui se pose après notre analyse serait, une fois étudiées et comparées les spécificités des communautés nationales, de rendre compte d'un savoir commun. Celle pourrait conduire à une « diplomatie scientifique » franco-brésilienne qui nous permettrait de comprendre ce qui reste, encore aujourd'hui, du savoir tacite. Nous reprenons ici la pensée du professeur Bernard Lamizet ${ }^{20}$, qui explique que la science a un temps court et long. Pour lui le temps de la science est une médiation entre un temps court de l'activité sociale des chercheurs, structurée par des événements et par des confrontations et de leur subjectivité structurée par le temps de leur vie personnelle et un temps long de la science, qui est celui de son histoire et des grandes dynamiques, dont on ne peut avoir l'expérience, mais que l'on ne peut que savoir (Lamizet 2010 : 174).

Enfin, nous ne pouvons que remarquer et saluer le travail qui commence aussi à en niveau international: les toutes récentes initiatives d'une « coprésence » franco-brésilienne aux numéros 51 et 52 de la revue française de Communication E' Organisation, ainsi qu'au Séminaire International de

20 Bernard Lamizet est professeur émérite de Sciences de l'information et de la communication à l'Institut d'études politiques de Lyon. Ses activités de recherches et ses publications portent sur la sémiotique, l'esthétique, la sémiotique politique, les politiques urbaines et la théorie de la communication. 
Communication Organisationnelle - SICO, réalisé tous les deux ans dans l'État de Minas Gerais au Brésil, avec la production d'un livre e-book (Marques et al. 2017).

\section{BIBLIOGRAPHIE}

BOURE Robert, 2007, Les sciences humaines et sociales en France: une approche historique, collection «Échanges », Cortil-Wodon : Éditions modulaires européennes.

CORDELIER, Benoit et Hélène MONTAGNAC-MARIE, 2008, "Auteurs", Dans Conduire le changement organisationnel? $\mathrm{n}^{\circ}$ 33. Communication \& Organisation. https://communicationorganisation.revues.org/33

ALMEIDA d', Nicole et Yanita ANDONOVA, 2006, " La Communication des Organisations", dans Sciences de l'information et de la communication: objets, savoirs, discipline. Olivesi Stéphane. La communication en plus. Grenoble: Presses Univ. de Grenoble.

DELCAMBRE Pierre, "Faire un état des recherches en " com. des organisations ", 2008. http://dolanca.free.fr/SIC/09SIC.pdf

GALLEZOT Gabriel, 2003, "ArchiveSIC, Archive Ouverte en Sciences de l'Information et de la Communication: rôle, fonctionnement et usage.», Revue professionnelle.

KUNSCH Margarida, 2006, "Comunicação organizacional: complexidade e atualidade», Revistas Univerciencia: Novos Olhares, no 18, 23-31.

LAMIZET Bernard, 2010, «Une nouvelle approche de l'épistémologie», dans Les savoirs communicants : entre histoire, usages et innovations, 167-74. Collection Sociétés. Dijon : Éditions universitaires de Dijon.

MARQUES Ângela et Ivone de LOURDES DE OLIVEIRA, 2015, «Configuration du champ de la Communication Organisationnelle au Brésil: problématisation, possibilités et potentialités ", Communication et organisation, $\mathrm{n}^{\circ}$ 48, 109-124. doi:10.4000/ communicationorganisation.5092

SALGUEIRO MARQUES Ângela, Ivone de LOURDES OLIVEIRA, Fabia LIMA et Daniel REIS, 2017, Comunicaşão Organizacional: Vertentes Conceituais e Metodológicas. Vol.1. III SICO Seminario Internacional de Comunicação Organizacional. Belo Horizonte: PPGCOM UFMG. https://drive.google.com/file/ d/0B1GC0XIYJfiVUlZWOVptaDFldnc/view

PARIS R. S., Alice Zozima PARIS REGO DE SOUZA, 2017, «Le monde des Communications Organisationnelles : Pratiques et Recherches. Une étude généalogique, France et Brésil », Toulon.

PUTNAM Linda L., Nelson PHILLIPS et Pamela CHAPMAN, 1996, « Metaphors of Communication and Organisation ", dans Handbook of Organization Studies, Sterwart Clegg, Cynthia Hardy, and Walter Nord, 375-408. http://courses.washington.edu/ilis580/ readings/Putnam27.pdf

SAVIDAN Patrick et Sylvie MESURE, 2006, Dictionnaire des sciences humaines. French \& European Publications. Google-Books-ID : PdRzjwEACAAJ. 
Résumés: Cet article s'intéresse à la construction de communautés scientifiques en communication organisationnelle, en France et au Brésil. Les résultats d'une première étude montrent les décalages et les différences qui structurent l'institutionnalisation de ce champ de recherche dans les deux pays. Une seconde, analysant le jeu des références bibliographiques de chercheurs confirmés ces dix dernières années, montre les rares références aux collègues de l'autre pays. Dès lors nous pouvons dire que si quelques passeurs existent, si des organisateurs d'échanges se mobilisent actuellement, nous ne pouvons estimer qu'existerait déjà une communauté scientifique dans cette sousdiscipline : les socialisations des chercheurs sont encore trop éloignées.

Mots-clés : Communication Organisationnelle, France, Brésil, échanges, communauté scientifique franco-brésilienne.

Abstract: This article concerns to the construction of scientific communities in organizational communications in France and Brazil. The results of a first study shows the offsets and differences that structure the institutionalization of this field of research in both countries. A second study, that analyzes the set of bibliographic references of confirmed researchers in the last ten years, shows the rare references to colleagues from another country. Therefore, we can say that if some of them play the role of transmitter and if the organizers of exchanges mobilize more now, we can not estimate that there is already a scientific community in this sub-discipline: the socializations of the researchers are still very distant.

Keywords: Organizational Communication, France, Brazil, exchanges, Franco-Brazilian scientific community.

Português: Este artigo se interessa a constrụ̧ão de comunidades cientificicas em comunicação organizacional, na França e no Brasil. Os resultados de um primeiroestudo mostram as decalagens $e$ as diferenças que estruturam a institucionalização deste campo de pesquisa em ambos os países. Um segundo, analisando o conjunto de referências bibliográficas de pesquisadores confirmados nesses últimos dez anos, mostra as raras referências aos colegas de outro país. Portanto, nós podemos dizer que, se alguns fazem o papel de transmissor, se os organizadores de intercâmbio se mobilizarem mais atualmente, não podemos estimar que já exista uma comunidade científica nesta subdisciplina: as socializações dos pesquisadores ainda estão muito distantes.

Palavras-chave: Comunicação Organizacional, França, Brasil, intercâmbios, comunidade cientifica franco-brasileira. 